\title{
O status ecológico das comunidades de fungos coprófilos
}

\author{
Francisco J. Simões Calaça ${ }^{1,2}$, Jéssica Conceição Araújo ${ }^{2}$ \& Solange Xavier- \\ Santos $^{2}$
}

(1) Universidade de Brasília - campus Darcy Ribeiro, Instituto de Ciências Biológicas, Departamento de Ecologia, Laboratório de Ecologia de Ecossistemas, Asa Norte 70910-900, Brasília, Distrito Federal, Brasil. E-mail: calacafjs@gmail.com

(2) Universidade Estadual de Goiás - campus de Ciências Exatas e Tecnológicas, Laboratório de Biodiversidade do Cerrado, Rodovia BR 153, no 3105, Fazenda Barreiro do Meio, Anápolis 75132-400, Goiás, Brasil. E-mail: jessmycologist@gmail.com, solxav@yahoo.com.br

Calaça F.J.S., Araújo J.C. \& Xavier-Santos S. (2017) O status ecológico das comunidades de fungos coprófilos. Pesquisa e Ensino em Ciências Exatas e da Natureza, 1(2): 136-143.

Resumo: Buscamos discutir o status ecológico das comunidades de fungos coprófilos lato sensu, apresentando pontos chave para a formulação de hipóteses e futuros estudos que visem entender os fatores ecológicos que modulam a escolha da vida coprófila por estes fungos. Apresentamos um novo termo (copromicodiversidade), que melhor designa a amplitude do grupo, considerando a total abrangência do mesmo, incluindo a diversidade morfológica, funcional e ecológica de uma dada população de fungos coprófilos registrados em uma região geográfica específica. Além disso, levantamos questões relacionadas ao ciclo de vida destes organismos, considerando recentes estudos bem como a premissa tradicionalmente aceita que defende a necessidade dos esporos de fungos coprófilos passarem pelo trato digestivo dos animais, para sua posterior emergência nas fezes. Esperamos que futuras pesquisas possam melhor definir estas comunidades, evitando incertezas sobre a definição do estilo de vida coprófilo, mesmo que algumas espécies possam crescer em outros substratos (fimícolas), mas ainda apresentando relações com hospedeiros animais (coprofilia). Enquanto esperamos por direções futuras, novas hipóteses devem ser planejadas e testadas visando os aspectos que verdadeiramente modulam a ocorrência de fungos coprófilos em diferentes ambientes.

Palavras chave: Copromicodiversidade, ecologia fúngica, escolha de substratos, fungos de esterco.

\section{The ecological status of coprophilous fungi communities}

Abstract: We discuss the ecological status of coprophilous fungi communities lato sensu, presenting key points to the definition of scientific hypothesis and future studies aiming to understand the ecological factors that modulate the coprophilous lifestyle's choice by these fungi. We present a new scientific term (copromycodiversity) that better describes the magnitude of this group, considering the comprehensiveness of this fungal group, including the morphological, physiological and ecological diversities to a coprophilous fungi population recorded to a specific geographical region. Furthermore, we raised some questions related to the life cycle of these fungi, taking into account recent studies as well as the traditionally accepted assumption that supports the spore passage through animals's gut to its growth and development on dung. We expect that future research would best define these communities, avoiding uncertainties about the definition of the coprophilous lifestyle, even that some species would growth on others substrates (fimicolous fungi), but still displaying some connection with its animal host (coprophilia). While we are waiting for future directions, new hypothesis must be planned and tested aiming the predictors that truly modulates the occurrence of coprophilous fungi in different environments.

Key words: Copromycodiversity, dung fungi, fungal ecology, substrate choice. 
Fungos coprófilos, comumente denominados de fungos do esterco, são um diverso grupo de organismos especializados em crescer e viver sobre fezes de diversas espécies de animais, sejam estes herbívoros, carnívoros ou onívoros (Lundqvist 1972; Bell 1983). No entanto, a escolha da vida coprófila requer muitas adaptações para garantir a sobrevivência nestes substratos consideravelmente extremos, devido à, por exemplo, sua composição e a competição entre diversas espécies de organismos ali presentes (e.g., bactérias, insetos coprófagos). Por causa disso, fungos coprófilos (e/ou fimícolas) apresentam adaptações ecológicas e fisiológicas relatadas amplamente na literatura (Lundqvist 1972; Bell 1983; Richardson 2001a). As denominações coprófilo e fimícola também apresentam significados distintos. Definem-se como fungos coprófilos aqueles que, até o que se conhece de sua ecologia, crescem apenas sobre fezes. Fímicolas são aqueles fungos generalistas, uma vez que podem se desenvolver sobre uma miscelânea de substratos. Embora estas terminações facilitem o entendimento acerca da escolha do substrato destes fungos, muitos autores referem-se a estes organismos apenas como fungos coprófilos.

Estes fungos podem, ainda, ser classificados em dois grupos: fungos coprófilos stricto e lato sensu. Fungos coprófilos stricto sensu são aqueles taxonomicamente pertencentes aos filos Ascomycota, Basidiomycota e Mucoromycota (Hibbett et al. 2007; Spatafora et al. 2016). A denominação lato sensu, refere-se aos grupos de organismos que não pertencem ao Reino Fungi, mas que por tradição, são estudados na micologia, como os mixomicetos (Amebozoa) e mixobactérias (Proteobacteria), sendo denominados de pseudofungos (Reichenbach 1999; Calaça \& Xavier-Santos 2012; Eliasson 2012; Cavalier-Smith et al. 2015). Dentre os pseudofungos, são considerados coprófilos (ou fimícolas) apenas aqueles com a capacidade de crescerem sobre fezes. Para algumas espécies de mixomicetos, notoriamente, há registros apenas sobre fezes, o que confirma, até então, sua escolha pela vida coprófila (Eliasson et al. 1991; Eliasson \& Keller 1999; Novozhilov \& Schnittler 2000; Eliasson 2012). Em nossos estudos sobre ecologia e diversidade de fungos coprófilos, incluímos também os pseudofungos como objeto de trabalho. Desta forma, como no presente texto, consideramos fungos coprófilos, todos aqueles que desenvolvem parte de seu ciclo de vida, seja de forma obrigatória ou facultativa, sobre fezes.

O principal papel ecossistêmico deste grupo está em sua participação na ciclagem da energia egestada pelos animais nas fezes, ciclando os nutrientes não absorvidos e decompondo este substrato no ambiente. Os dados até então publicados sobre fungos coprófilos, têm nos permitido identificar padrões ecológicos das comunidades fúngicas habitantes do esterco. Dentre estas particularidades, destacam-se as diferenças nas escolhas de substratos (i.e., espécies que, aparentemente, preferem fezes de herbívoros ou carnívoros), sazonalidade ou padrões de ocorrência influenciados pela altitude ou pela própria comunidade hospedeira (Richardson 2001a, 2015). Como observado por Delpont (comunicação pessoal, 2017), alguns animais que vivem fora de sua região natural podem permitir o aparecimento de certos fungos que, usualmente, são associados com estes animais e suas regiões geográficas de origem, desde que as condições ambientais (e.g., clima) sejam favoráveis.

$\mathrm{Na}$ micologia de fungos coprófilos, há a premissa tradicionalmente aceita que defende a necessidade dos esporos de fungos coprófilos passarem pelo trato digestivo dos animais, para sua posterior emergência nas fezes (Lundqvist 1972), que ocorre após a quebra da dormência dos esporos por meio dos efeitos químicos, térmicos e mecânicos do trato digestivo dos animais (Bell 1983, 2005; Richardson 2001a). Esta teoria é aceita pelos estudiosos deste grupo, principalmente aqueles dedicados à ecologia do grupo. Contudo, recentemente, Newcombe et al. (2016) apresentaram uma revisão do ciclo de vida dos fungos coprófilos. Baseado especialmente em estudos com Sordaria fimicola (Roberge ex Desm.) Ces. \& De Not. (Sordariaceae: Ascomycota), o artigo defende um estilo de vida coprófilo facultativo, ao invés da obrigatoriedade da associação com o hospedeiro animal, como conhecido até então.

Em seu trabalho, Newcombe et al. (2016) avaliaram se o crescimento e fecundidade da gramínea Bromus tectorum L. (Poaceae), bem como se a escolha do animal em forragear esta planta poderia ser afetada por fungos coprófilos. Os autores utilizaram três táxons de fungos coprófilos: Sordaria fimicola, Preussia sp. e Sporormiella sp., que são caracterizadas como 
espécies fimícolas. Sordaria fimicola, por exemplo, tem sido registrada ocorrendo em solo, resíduos vegetais diversos, papel e tecidos têxteis (Lundqvist 1972). Por outro lado, os gêneros Preussia e Sporormiella são considerados sinônimos, uma vez que estudos moleculares não suportaram diferenças filogenéticas entre ambos (Kruys \& Wedin 2009; Mapperson et al. 2014). Em adição, espécies desses gêneros também podem crescer e completar seu ciclo de vida em outros substratos, notoriamente solo e resíduos vegetais (Cain 1961; Dugan et al. 1995; Guarro et al. 1997; Gonzalez-Menendez et al. 2017).

Agora, parece que nossa compreensão sobre "o que determina quais fungos são realmente coprófilos?" deve ser revisitada, com o objetivo de entender fatores intrínsecos que permitem a adaptação e necessidade da vida coprófila, bem como a coexistência com os animais hospedeiros dos esporos. Como mencionado por Newcombe et al. (2016), as espécies utilizadas no estudo não são obrigatoriamente coprófilas. Assim, se torna necessário novos estudos com outros gêneros de fungos coprófilos e espécies de plantas. Pilobolus spp., Hypocopra spp., Zygopleurage spp. e Saccobolus spp., por exemplo, são gêneros bastante indicativos do estilo de vida coprófilo e da real dependência da passagem pelo trato digestivo animal para posterior emergência do esporo.

Devido a características das comunidades de fungos coprófilos e animais hospedeiros, as populações de fungos encontradas, por exemplo, em amostras fossilizadas de fezes, podem refletir a estrutura populacional da megafauna pleistocênica, como demonstrado por Davis (1987) e, recentemente, por Basumatary \& McDonald (2017) e van Asperen (2017). Embora estes estudos abordem a ocorrência de espécies principalmente fimícolas, seu declínio no passado pode estar associado à redução de hospedeiros, provavelmente porque estas espécies, estocasticamente, associaram-se com o trato digestivo de seus hospedeiros ao invés da escolha por outros substratos (e.g., resíduos vegetais ou a escolha da vida endofítica). Entender o porquê de diferentes padrões na história de vida desses fungos é um passo que ainda precisa ser elucidado.

A ecologia dos fungos de esterco é influenciada por diferentes aspectos. Estes vão estruturar as características físico-químicas do esterco ( $\mathrm{CfqE}$ ) e, consequentemente, as comunidades que vão se desenvolver neste substrato (Lundqvist 1972; Richardson 2001a). CfqE são moduladas pela diversidade animal. Uma grande heterogeneidade de espécies animais implica em grande diversidade de tipos e de escolhas de substratos. Lundqvist (1972) apresentou uma interessante abordagem sobre as preferências de substratos para diversas espécies de fungos coprófilos. Recentes estudos já demonstraram como a composição e estequiometria das fezes influenciam e modulam a comunidade fúngica (e.g., Richardson 2001a).

Se a heterogeneidade animal influencia a estequiometria e teores de nutrientes disponíveis nas fezes, o ambiente é, portanto, o primeiro direcionador das $\mathrm{CfqE}$, uma vez que este modula a composição da fauna, as interações ecológicas que ocorrem neste microcosmo (e.g., predação de fungos por artrópodes, competição por nutrientes entre fungos e bactérias) e mesmo a duração do esterco como substrato disponível. As pesquisas sobre comunidades de fungos coprófilos lato sensu tem aumentado nos últimos anos, principalmente em países da Europa, África, Ásia e América do Sul. Alguns registros sobre a distribuição de espécies foram publicadas por Eliasson (2012), Calaça et al. (2014), Abdel-Azeem \& Salem-Fatma (2015) e Richardson (2015).

No Brasil, os estudos focados na biodiversidade de fungos coprófilos têm seu registro mais antigo em uma publicação do micologista e botânico Carlos L. Spegazzini (1858-1926), que publicou o primeiro registro de um fungo coprófilo para o Brasil: Psilocybe merdaria (Fr.) Ricken (Katinas et al. 2000). Posteriormente, como apresentado por Calaça et al. (2014), diversos registros ocasionais foram publicados, sendo o primeiro estudo com foco em taxonomia e diversidade restrito apenas ao grupo publicado em 2001, pelo micologista Michael J. Richardson, em visita ao Brasil (Richardson 2001b). A partir de 2010, novos estudos foram conduzidos no país, elevando o número de espécies de fungos coprófilos lato sensu. Até 2013, havia o registro de ocorrência de 210 espécies de fungos coprófilos lato sensu no país, distribuídas em $44 \%$ dos estados brasileiros. Em 2016, o total de espécies conhecidas para o país elevou-se para 225 
(Calaça \& Xavier-Santos 2016). Esses dados mostram que, estimulando novos esforços em áreas sub exploradas ou mesmo regiões sem registros de fungos coprófilos, muito mais espécies podem ser registradas, contribuindo para a expansão do conhecimento da diversidade deste grupo no país e, consequentemente, no mundo.

Como destacado por Calaça et al. (2014), a riqueza de fungos coprófilos lato sensu no Brasil é alta, ainda que comparada a dados sobre essa diversidade para outras localidades do globo (Richardson 2015). Embora ainda existam poucos micologistas dedicados a este grupo no país, o número de espécies tende a crescer, como demonstrado por estudos recentes (Calaça \& Xavier-Santos 2016), o que reforça a necessidade da formação de recursos humanos que busquem avaliar a biodiversidade do grupo, bem como seu potencial biotecnológico. A Tabela 1 mostra um panorama da riqueza dos taxa de fungos coprófilos lato sensu para o Brasil até o ano de 2016, com base nos dados apresentados por Calaça et al. (2014) e Calaça \& Xavier-Santos (2016). Estes dados corroboram a afirmativa sobre o potencial da biodiversidade de espécies de fungos no país (Maia et al. 2015), especialmente quando consideramos para estudo, hábitats potenciais mas, muitas vezes, erroneamente menosprezados.

Tabela 1. Panorama da riqueza observada para os diferentes taxa de fungos coprófilos (lato sensu) conhecidos para o Brasil até o ano de $2016^{1}$, de acordo com Calaça et al. (2014) e Calaça \& Xavier-Santos (2016).

\begin{tabular}{|c|c|c|c|}
\hline \multicolumn{3}{|c|}{ Taxa } & \multirow{2}{*}{$N^{0}$ de espécies } \\
\hline \multirow{20}{*}{ 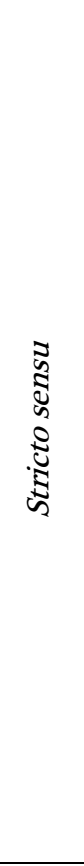 } & Filo & Ordem & \\
\hline & Mucoromycota & Mucorales & 54 \\
\hline & \multirow{14}{*}{ Ascomycota } & Capnodiales & 1 \\
\hline & & Dothideales & 1 \\
\hline & & Eurotiales & 12 \\
\hline & & Glomerellales & 1 \\
\hline & & Hypocreales & 8 \\
\hline & & Pyxidiophorales* & 1 \\
\hline & & Microascales & 3 \\
\hline & & Pezizales* & 29 \\
\hline & & Phomatosporales & 1 \\
\hline & & Pleosporales* & 6 \\
\hline & & Saccharomycetales & 22 \\
\hline & & Sordariales* & 39 \\
\hline & & Thelebolales* & 8 \\
\hline & & Xylariales* & 2 \\
\hline & \multirow{4}{*}{ Basidiomycota } & Agaricales & 18 \\
\hline & & Sporidiobolales & 4 \\
\hline & & Tremellales & 1 \\
\hline & & Trichosporonales & 1 \\
\hline \multirow{6}{*}{ 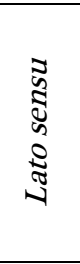 } & \multirow{4}{*}{ Myxomycota } & Liceales & 4 \\
\hline & & Physarales & 2 \\
\hline & & Stemonitales & 1 \\
\hline & & Trichiales & 4 \\
\hline & Proteobacteria & Myxococcales & 1 \\
\hline & Oomycota & Pythiales & 1 \\
\hline Total & 6 & 25 & 225 \\
\hline
\end{tabular}

${ }^{1}$ Ordens com adições de espécies após 2013 são marcadas com um asterisco $(*)$.

A Micologia como ciência, por si só, foi objeto de incerteza dentro das ciências biológicas, sendo estudada dentro da botânica e inclusa em muitos livros-texto botânicos (e.g., Raven et al. 2007). Mesmo dentro da Micologia, há inúmeras subdivisões e grupos de estudos. Considerando a grande heterogeneidade dos fungos coprófilos, torna-se necessário, assim como para os demais grupos de fungos, a proposição de um novo termo que melhor descreva toda a 
amplitude deste grupo. O termo copromicodiversidade está sendo proposto aqui, baseado nos termos gregos kópros (esterco) e mýkēs (fungo) e do termo latino diversitate (diversidade). Portanto, copromicodiversidade refere-se à total abrangência deste grupo, incluindo a diversidade morfológica, funcional e ecológica de uma dada população (copromicobiota, do grego bíos, vida) de fungos coprófilos registrados em uma região geográfica específica.

Esperamos que futuras pesquisas possam melhor definir estas comunidades, evitando incertezas sobre a definição do estilo de vida coprófilo, mesmo que algumas espécies possam crescer em outros substratos, mas não escapando da relação com a coprofilia. Como discutido, muitas interações modulam a ocorrência de fungos coprófilos (Figura 1). Ainda, diversos estudos já demonstraram que espécies verdadeiramente coprófilas necessitam da passagem através do trato digestivo para a emergência dos esporos e posterior frutificação (e.g., Lundqvist 1972; Bell 1983, 2005; Doveri 2004, 2017; Calaça \& Xavier-Santos 2016). Ecologia, diversidade, traços funcionais e fisiologia, determinam como a copromicobiota irá se expressar. Finalmente, estas interações vão determinar como a decomposição e ciclagem de nutrientes do esterco irão ocorrer nos ecossistemas. Enquanto esperamos por direções futuras, novas hipóteses devem ser delineadas e testadas, visando os aspectos que verdadeiramente modulam a ocorrência de fungos coprófilos em diferentes ambientes.

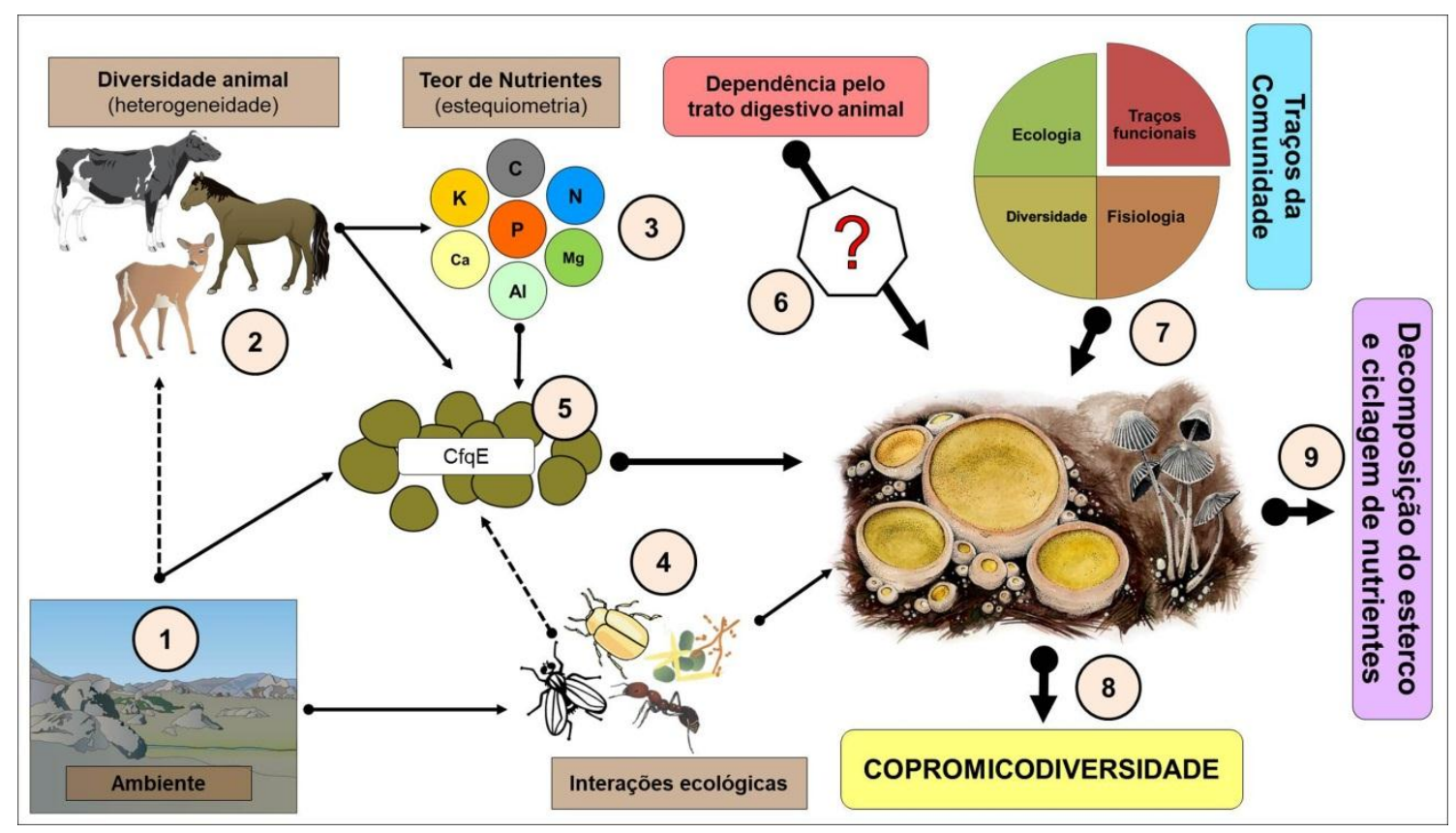

Figura 1. Mapa conceitual das interações que modulam a copromicodiversidade em diferentes ambientes. Setas pontilhadas representam interações direcionadoras indiretas; setas inteiras representam efeitos diretos. A espessura das setas indica o quão representativo é o efeito como direcionador do próximo processo. (1) $\mathrm{O}$ ambiente é um direcionador indireto da diversidade de espécies animais, pois diferentes características bióticas e abióticas determinam a ocorrência, traços funcionais e ecologia de cada animal. Por outro lado, o ambiente representa um efeito direto sobre as características físico químicas do esterco (CfqE), duração do mesmo como um substrato disponível e interações ecológicas no próprio esterco (4). (23) A dieta animal determina a composição do esterco (estequiometria, CfqE) e a escolha de substratos pelos indivíduos de diferentes espécies fúngicas, sendo portanto um efeito direto para a estrutura da comunidade de fungos coprófilos no esterco (5). Para fungos coprófilos, a preferencia do tipo de hospedeiro, necessidade da passagem pelo trato digestivo (6) e traços da comunidade (7) determinam como a copromicodiversidade é expressa em cada ambiente (8), finalizando na decomposição do esterco e ciclagem dos nutrientes (9). Figura elaborada pelos autores, com vetores disponibilizados em "Integration and Application Network, University of Maryland Center for Environmental Science” (http://ian.umces.edu/symbols/). Ilustração da copromicodiversidade por Martin Makarewicz. 


\section{Agradecimentos}

Agradecemos a Michel Delpont pelos comentários e sugestões na versão prévia do manuscrito. A equipe editorial da Revista PECEN, pelas valorosas contribuições durante o processo de revisão. Aos avaliadores anônimos pela revisão crítica do manuscrito.

\section{Referências}

Abdel-Azeem A.M. \& Salem F.M. (2015) Fungi fimicola Aegyptiaci: I. Recent investigations and conservation in arid South Sinai. Mycosphere, 6(2): 174-194. doi: 10.5943/mycosphere/6/2/8

Basumatary S.K. \& McDonald H.G. (2017) Coprophilous fungi from dung of the Greater OneHorned Rhino in Kaziranga National Park, India and its implication to paleoherbivory and paleoecology. Quaternary Research, 88: 14-22. doi: 10.1017/qua.2017.34

Bell A. (1983) Dung Fungi: an illustrated guide to coprophilous fungi in New Zealand. New Zealand: Victoria University Press. 88 p.

Bell A. (2005) An illustrated guide to the coprophilous Ascomycetes of Australia. CBS Biodiversity Series 3. Utrecht: Centraalbureau voor Schimmelcultures. $172 \mathrm{p}$.

Cain R.F. (1961) Studies of coprophilous ascomycetes. VII. Preussia. Canadian Journal of Botany, 39: 1633-1666. doi: 10.1139/b61-144

Calaça F.J.S. \& Xavier-Santos S. (2012) Fezes de Herbívoros: um microcosmo inexplorado da diversidade fúngica. Heringeriana, 6(1): 52-55.

Calaça F.J.S. \& Xavier-Santos S. (2016) New records of coprophilous ascomycetes (Fungi: Ascomycota) from Brazil and Neotropical Region. Check List, 12(6): 2009, 1-9. http://dx.doi.org/10.15560/12.6.2009

Calaça F.J.S., Silva N.C. \& Xavier-Santos S. (2014) A checklist of coprophilous fungi and other fungi recorded on dung from Brazil. Mycotaxon, 128: 205. 1-22. doi: http://dx.doi.org/10.524 $8 / 128.205$

Cavalier-Smith T., Fiore-Donno A.M., Chao E., Kudryavtsev A., Berney C., Snell E.A. \& Lewis R. (2015) Multigene phylogeny resolves deep branching of Amoebozoa. Molecular Phylogenetics and Evolution, 83: 293-304. doi: 10.1016/j.ympev.2014.08.011

Davis O.K. (1987) Spores of the dung fungus Sporormiella: increased abundance in historic sediments and before Pleistocene Megafaunal Extinction. Quaternary Research, 28: 290-294. https://doi.org/10.1016/0033-5894(87)90067-6

Doveri F. (2004) Fungi Fimicoli Italici. Trento, A.M.B: Fondazione Studi Micologici. 1104 p.

Doveri F. (2017) Additional reports on coprophilous Lasiosphaeriaceae from tropical climates. Ascomycete.org, 9(2): 32-58.

Dugan F.M., Roberts R.G. \& Hanlin R.T. (1995) New and rare fungi from cherry fruits. Mycologia, 87: 713-718. http://dx.doi.org/10.2307/3760817

Eliasson U.H. (2012) Coprophilous Myxomycetes: Recent advances and future research directions. Fungal Diversity, 59(1): 85-90. http://dx.doi.org/10.1007/s13225-012-0185-6

Eliasson U.H. \& Keller H.W. (1999) Coprophilous myxomycetes: updated summary, key to species, and taxonomic observations on Trichia brunnea, Arcyria elaterensis, and Arcyria stipata. Karstenia, 39: 1-10.

Eliasson U.H., Keller H.W. \& Schoknecht J.D. (1991) Kelleromyxa, a new generic name for Licea fimicola (Myxomycetes). Mycological Research, 95: 1201-1207. doi: 10.1016/S0953-7562(09) $80011-7$

Gonzalez-Menendez V., Martin J., Siles J.A., Gonzales-Tejero M.R., Reyes F., Platas G., Tormo J.R. \& Genilloud O. (2017) Biodiversity and chemotaxonomy of Preussia isolates from the Iberian Peninsula. Mycological Progress, 16: 713-728. http://dx.doi.org/10.1007/s11557-017-1305-1

Guarro J., Abdullah S.K., Gene J. \& Al-Saadoon A.H. (1997) A new species of Preussia from submerged plant debris. Mycological Research, 101: 305-308. http://dx.doi.org/10.1017/S095 3756296002638 
Hibbett D.S., Binder M., Bischoff J.F., Blackwell M., Cannon P.F., Eriksson O.E., Huhndorf S., James T., Kirk P.M., Lücking R., Thorsten-Lumbsch H., Lutzoni F., Matheny P.B., McLaughlin D.J., Powell M.J., Redhead S., Schoch C.L., Spatafora J.W., Stalpers J.A., Vilgalys R., Aime M.C., Aptroot A., Bauer R., Begerow D., Benny G.L., Castlebury L.A., Crous P.W., Dai Y.G., Gams W., Geiser D.M., Griffith G.W., Gueidan C., Hawksworth D.L., Hestmark G., Hosaka K., Humber R.A., Hyde K.D., Ironside J.E., Kõljalg U., Kurtzman C.P., Larsson K.H., Lichtwardt R., Longcore J., Miadlikowska J., Miller A., Moncalvo J.M., Mozley-Standridge S., Oberwinkler F., Parmasto E., Reeb V., Rogers J.D., Roux C., Ryvarden L., Sampaio J.P., Schüssler A., Sugiyama J., Thorn R.G., Tibell L., Untereiner W.A., Walker C., Wang Z., Weir A., Weiss M., White M.M., Winka K., Yao Y.J. \& Zhang N. (2007) A higher-level phylogenetic classification of the Fungi. Mycological Research, 111: 509-547. doi: 10.1016/j.mycres.2007.03.004

Katinas L., Gutiérrez D.G. \& Robles S.S.T. (2000) Carlos Spegazzini (1858-1926): Travels and botanical work on vascular plants. Annals of the Missouri Botanical Garden, 87: 183-202.

Kruys Å. \& Wedin M. (2009) Phylogenetic relationships and an assessment of traditionally used taxonomic characters in the Sporormiaceae (Pleosporales, Dothideomycetes, ascomycota), utilising multi-gene phylogenies. Systematics and Biodiversity, 7: 465-478. doi: 10.1017/S1477 200009990119

Lundqvist N. (1972) Nordic Sordariaceae s. lat. Symbolae Botanicae Upsalienses, 20(1): 1-314.

Maia L.C., Carvalho-Júnior A.A., Cavalcanti L.H., Gugliotta A.M., Drechsler-Santos E.R., Santiago A.L.C.M.A., Cáceres M.E.S., Gibertoni T.B., Aptroot A., Giachini A.J., Soares A.M.S., Silva A.C.G., Magnago A.C., Goto B.T., Lira C.R.S., Montoya C.A.S., Pires-Zottarelli C.L.A., Silva D.K.A., Soares D.J., Rezende D.H.C., Luz E.D.M.N., Gumboski E.L., Wartchow F., Karstedt F., Freire F.M., Coutinho F.P., Melo G.S.N., Sotão H.M.P., Baseia I.G., Pereira J., Oliveira J.J.S., Souza J.F., Bezerra J.L., Araujo-Neta L.S., Pfenning L.H., Gusmão L.F.P., Neves M.A., Capelari M., Jaeger M.C.W., Pulgarín M.P., Menolli-Junior N., Medeiros P.S., Friedrich R.C.S., Chikowski R.S., Pires R.M., Melo R.F., Silveira R.M.B., Urrea-Valencia S., Cortez V.G. \& Silva V.F. (2015) Diversity of Brazilian Fungi. Rodriguésia, 66: 1033-1045. http://dx.doi.org/10.1590/21757860201566407

Mapperson R.R., Kotiw M., Davis T.A. \& Dearnaley J.D.W. (2014) The diversity and antimicrobial activity of Preussia sp. endophytes isolated from Australian dry rainforests. Current Microbiology, 68: 30-37. http://dx.doi.org/10.1007/s00284-013-0415-5

Newcombe G., Campbell J., Griffith D., Baynes M., Launchbaugh K. \& Pendleton R. (2016) Revisiting the life cycle of dung fungi, including Sordaria fimicola. PLoS ONE, 11(2): e0147425. doi: 10.1371/journal.pone.0147425

Novozhilov Y.K. \& Schnittler M. (2000) A new coprophilous species of Perichaena (Myxomycetes) from the Russian Arctic (the Taimyr Peninsula and the Chukchi Peninsula). Karstenia, 40(1-2): 117-122.

Raven P.H., Evert R.F. \& Eichhorn S.E. (2007) Biology of Plants. $7^{\circ}$ edition. Arcata: W.H. Freeman \& Co. 944 p.

Reichenbach H. (1999) The ecology of the myxobacteria. Environmental Microbiology, 1(1): 1521. http://dx.doi.org/10.1046/j.1462-2920.1999.00016.x

Richardson M.J. (2001a) Diversity and occurrence of coprophilous fungi. Mycological Research, 105: 387-402. http://dx.doi.org/10.1017/S0953756201003884

Richardson M.J. (2001b) Coprophilous fungi from Brazil. Brazilian Archives of Biology and Technology, 44(3): 283-289. http://dx.doi.org/10.1590/S1516-89132001000300010

Richardson M.J. (2015) Records of coprophilous fungi - a data set. Mycotaxon, 130: 925, 1-5. http://dx.doi.org/10.5248130.925

Spatafora J.W., Chang Y., Benny G.L., Lazarus K., Smith M.E., Berbee M.L., Bonito G., Corradi N., Grigoriev I., Gryganskyi A., James T.Y., O’Donnell K., Roberson R.W., Taylor T.N., Uehling J., Vilgalys R., White M.M. \& Stajich J.E. (2016) A phylum-level phylogenetic classification of zygomycete fungi based on genome-scale data. Mycologia, 108: 1028-1046. http://dx.doi.org/1 0.3852/16-042 
van Asperen E.N. (2017) Fungal diversity on dung of tropical animals in temperate environments: Implications for reconstructing past megafaunal populations. Fungal Ecology, 28: 25-32. doi: 10.1016/j.funeco.2016.12.006 